\title{
Effects of the regulation of polysialyltransferase ST8SiaII on the invasiveness and metastasis of small cell lung cancer cells
}

\author{
LIANG GONG, XIANGDONG ZHOU, JINGXIANG YANG, YONGYUAN JIANG and HEPING YANG \\ Department of Respiratory Diseases, Southwest Hospital of the Third Military Medical University, \\ Shapingba, Chongqing 400038, P.R. China
}

Received May 18, 2016; Accepted June 30, 2016

DOI: $10.3892 /$ or.2016.5279

\begin{abstract}
Invasiveness and metastasis may seriously affect the prognosis of small cell lung cancer (SCLC). In the present study, we analyzed the effects and inherent mechanisms of action of polysialic acid-modified neural cell adhesion molecule (NCAM) on the invasive and metastatic potential of SCLC. Gene transfection and short hairpin RNA (shRNA) interference were used to enhance or inhibit, respectively, the expression of polysialyltransferase ST8SiaII in the SCLC cell line H446. We studied in vitro positive or negative changes in the invasive and metastatic potential of the SCLC cells as well as the changes in expression of genes related to signaling molecules and metastasis. When ST8SiaII expression was enhanced, the in vitro transmembrane invasion $(\mathrm{P}<0.01)$ and migration $(\mathrm{P}<0.01)$ abilities of the SCLC cells markedly increased. Phosphorylation levels of fibroblast growth factor receptor 1 (FGFR1), extracellular signal-related kinase 1/2 (ERK1/2), and matrix metalloproteinase-9 (MMP-9) in the SCLC cells were also significantly increased. In contrast, when ST8SiaII expression was inhibited, the transmembrane invasion $(\mathrm{P}<0.01)$ and migration $(\mathrm{P}<0.01)$ of the SCLC cells as well as expression of the above signaling molecules were suppressed. Polysialic acid-modified NCAM on the surface of SCLC cells is closely related to the metastatic potential of these cells; regulation of ST8SiaII may thus affect the invasiveness and metastasis of SCLC, and these processes may be associated with phosphorylation of FGFR1, ERK1/2 or MMP-9.
\end{abstract}

\section{Introduction}

Lung cancer has become the world's leading cause of cancerrelated deaths; the 5-year survival rate of patients with small

Correspondence to: Dr Heping Yang, Department of Respiratory Diseases, Southwest Hospital of the Third Military Medical University, 30 Gaotanyan Street, Shapingba, Chongqing 400038, P.R. China E-mail: hepingyangcn@163.com

Key words: tumor metastasis, small cell lung cancer, polysialyltransferase, neural cell adhesion molecule, glycosylation cell lung cancer (SCLC), in particular, remains less than $15 \%$. High aggressiveness and a high metastatic potential are key features of SCLC and are the main causes of treatment failure among patients with SCLC $(1,2)$. Although SCLC is sensitive to chemotherapy and radiotherapy, most patients show only a partial response (PR), and few achieve complete remission (CR). In addition, the SCLC patients who do reach a PR or CR are known to be prone to relapse and metastasis within a short period $(3,4)$. Prevention of the invasiveness and metastasis of SCLC has become a bottleneck in the prognosis of SCLC patients and an urgent clinical problem (5).

Proteins or lipids on the cell surface are often linked to diverse sugar residues, thus, forming sugar chains with a variety of special structures that play an important role in the regulation of cellular functions; this process is known as glycosylation (6). Sialic acid (SA) is a nine-carbon monosaccharide that is added to the sugar chain ends during protein or lipid glycosylation and is covalently linked to the subterminal galactose $(\mathrm{Gal})$ or $\mathrm{N}$-acetylgalactosamine (N-GalNAc) via $\alpha-2,3, \alpha-2,6$ or $\alpha-2,8$ bonds. Polysialic acid (PSA) is a long polymer of sialic acid monosaccharide residues connected via $\alpha-2,8$ bonds (7). In mammals, the most important role of PSA is covalent modification of certain cell surface proteins, among which the most common is neural cell adhesion molecule (NCAM). PSA acts as a post-translational modification for these proteins and regulates their functions; its attachment is mainly catalyzed and completed by one of two polysialyltransferases: ST8SiaII or ST8SiaIV (8). Various studies showed that PSA is highly expressed during embryogenesis, but in healthy adults, its expression is low except in nerve tissues. Recent studies showed $(9,10)$ that re-expression of PSA and its transferases are detectable in certain tumors, such as melanoma, breast cancer, malignant Wilms' tumor, glioma, lung and colorectal cancer, medullary thyroid-like tumors and T-cell malignant lymphoma, among others, and that the expression of PSA-NCAM is closely related to the tumor progression and poor prognosis of the patients, particularly in SCLC, pancreatic cancer and neuroblastoma. However, the specific causes and mechanisms are not yet clear.

The aims of the present study were to modify the expression of polysialyltransferase within SCLC cells in culture to change the modification by PSA of cellular NCAM and to analyze its effects on the invasive and metastatic potential of SCLC cells (and the possible mechanisms). The ultimate aim 
was to lay a foundation for further exploration of the metastatic mechanism and development of new targeted modalities for prevention of SCLC.

\section{Materials and methods}

Culture and transfection of SCLC cells. Cells of the human SCLC cell line H446 were purchased from the Shanghai Institutes for Biological Sciences (Shanghai, China), then routinely cultured in RPMI-1640 medium (HyClone Laboratories, Inc., Logan, UT, USA) containing $10 \%$ fetal bovine serum (Gibco Life Technologies, Grand Island, NY, USA) at $37^{\circ} \mathrm{C}$ and $5 \% \mathrm{CO}_{2}$.

H446 cells were transfected with the ST8SiaII-encoding lentiviral expression vector and a recombinant plasmid (Sigma-Aldrich, St. Louis, MO, USA) that can intracellularly express short hairpin RNA (shRNA). The transfection-positive cells were then screened and used to establish the lung cancer cell line H446-ST8SiaII-LENTI that overexpress the polysialyltransferase ST8SiaII (ST8SiaII was previously abbreviated as STX; and ST8SiaIV was previously abbreviated as PST; these terms may cause confusion) as well as the H446-ST8SiaIIshRNA cell line that shows inhibited expression of ST8SiaII. The plasmid transfection reagent Lipofectamine 2000 was purchased from Invitrogen Life Technologies (Grand Island, NY, USA).

Immunofluorescence and confocal detection. Immunofluorescence analysis (IF) was performed to detect the expression of PSA-NCAM and polysialyltransferase within the SCLC H446 cells. The procedures were as follows: i) the cells were seeded in pretreated 12 -well plates for subculturing, and when the cells attained $\sim 70 \%$ confluency, the coverslip was removed and the cells were washed with phosphate-buffered saline (PBS) three times, for 5 min each time. ii) The cells were fixed in $4 \%$ paraformaldehyde for $15 \mathrm{~min}$, and then stored in PBS at $4^{\circ} \mathrm{C}$. iii) The cells were removed from the solution and washed with PBS three times, 5 min each; before the assay for polysialyltransferase, Tween-20 was added, and the cells were processed for $15 \mathrm{~min}$, and then washed with PBS three times, for 5 min each time. iv) Non-specific binding sites on the cells were blocked with $10 \%$ normal serum for 10 min. v) A mouse anti-human PSA-NCAM monoclonal antibody (mAb; diluted 1:300; LifeSpan BioSciences, Inc., Seattle, WA, USA) and rabbit anti-human anti-ST8SiaII mAb (diluted 1:300; Sigma-Aldrich) were added for the immunoassay, and the mixtures were incubated in a humidified chamber at $4^{\circ} \mathrm{C}$ overnight. vi) The cells were removed from these solutions and washed with PBS three times, 5 min each, after which the fluorescence-labeled immunoglobulin $\mathrm{G}(\mathrm{IgG})$ [green fluorescence staining with fluorescein isothiocyanate (FITC), Cy3 red fluorescence staining, diluted 1:200] was added with subsequent incubation for $1 \mathrm{~h}$ in the dark at room temperature, and 4',6-diamidino-2-phenylindole (DAPI) was used to stain the nuclei. Then, the cells were washed with PBS three times, for $5 \mathrm{~min}$ each time, followed by one rinse with distilled water. vii) The cells were mounted with $90 \%$ glycerol; an anti-quenching mounting liquid was added dropwise, and the cells were then examined and photographed under an ordinary fluorescence microscope or a laser scanning confocal microscope [LSCM, Leica TCS-SP5; Leica Application Suite Advanced Fluorescence (LAS AF) software].

$R T-P C R$. RT-PCR was utilized to analyze the mRNA expression of polysialyltransferases ST8SiaII and ST8SiaIV in the human SCLC H446 cells for further experimental screening. In order to detect the expression of ST8SiaII and ST8SiaIV in $\mathrm{H} 446$ cells, the following primers were designed $\left(5^{\prime} \rightarrow 3^{\prime}\right)$ : ST8SiaII-F, GGATCAGCAAGCAGGAGTATG and ST8SiaII-R, AGAAAGGGTGTAACGCAACTAA; ST8SiaIV-F, GAGATGTGTCAGTGGTCAAGAG and ST8SiaIV-R, GAAACTTCAGGTAGGAGGCTATG.

The procedures were as follows: i) according to the GenBank sequences, IDTDNA Network Software (http://www.idtdna. com) was used to design the forward and reverse RT-PCR primers for polysialyltransferase ST8SiaII, ST8SiaIV, NCAM1 and NCAM2. ii) Total RNA was extracted according to the kit instructions. iii) After treatment with DNase I, $2 \mu \mathrm{g}$ of RNA and avian myeloblastosis virus (AMV) reverse transcriptase were added into the reaction mixture for reverse transcription. iv) Forward and reverse primers for different genes were added, and the PCR reaction was run in PCR buffer. v) The PCR reaction involved 30 cycles, with the annealing temperature set to 56,59 or $63^{\circ} \mathrm{C}$. vi) The PCR products were subjected to gel electrophoresis in $1.5 \%$ agarose and ethidium bromide staining, with glyceraldehyde-3-phosphate dehydrogenase (GAPDH) as the internal reference. We used Quantity One software (Bio-Rad Laboratories, Inc., Hercules, CA, USA) for analysis of the gel images, and the relative densitometric data from the strips under study and GAPDH were then compared.

Western blotting. Western blot analysis was performed to analyze the expression of polysialyltransferase proteins in each group after the treatment. We analyzed the expression differences in such signaling proteins as fibroblast growth factor receptor (FGFR), phospholipase C $\gamma 1$ (PLC $\gamma 1)$, and mitogen-activated protein kinase (MAPK) [extracellular signal-related kinase 1/2 (ERK1/2)], and their phosphorylation levels as well as the differences in expression of blood metastasis-related genes, such as matrix metalloproteinase-9 $(M M P-9)$ and vascular endothelial growth factor (VEGF). A mouse anti-human FGFR1 mAb, anti-FGFR1 (phospho-Y766) $\mathrm{mAb}$, anti-PLC $\gamma 1 \mathrm{mAb}$, anti-MMP-9 $\mathrm{mAb}$ and an anti-VEGF $\mathrm{mAb}$ were all purchased from Abcam Co. (Cambridge, UK); a rabbit anti-human phospho-PLC $\gamma 1$ (Ser1248) mAb, mouse anti-human p44/42 MAPK (Erk1/2) mAb, and a rabbit anti-phospho-p44/42 MAPK (Erk1/2) mAb were acquired from the Cell Signaling Technology, Inc. (Danvers, MA, USA). The following are the specific procedures. i) The cells were subdivided into the following groups: a, the H446 group; b, the 'ST8SiaII is overexpressed', group H446-ST8SiaII-LENTI; and c, the 'ST8SiaII expression is inhibited' group, H446-ST8SiaII-shRNA. ii) Total cellular protein of each group was extracted according to the kit instructions, and the Bradford method was used to test the protein concentrations. iii) A $25-\mu \mathrm{g}$ protein sample from each group (sample concentration $2 \mu \mathrm{g} / \mu \mathrm{l}$ ) was then subjected to sodium dodecyl sulfate-polyacrylamide gel electrophoresis (SDS-PAGE) on a $12 \%$ gel, at $100 \mathrm{~V}$ for $1.5 \mathrm{~h}$. iv) The proteins were then transferred to a polyvinylidene fluoride (PVDF) 
membrane, at $300 \mathrm{~mA}$ for $1 \mathrm{~h}$. v) The gel was discarded, and the membrane was washed with distilled water, after which the western blocking solution was added, and the membrane was slowly rocked on a shaker for blocking for $60 \mathrm{~min}$ at room temperature. Then, antibodies to ST8SiaII, FGFR, PLC $\gamma 1$, MAPK (ERK 1/2), MMP-9, VEGF or to the phosphorylated forms of the related proteins (diluted 1:1,000) were added for overnight incubation at $4^{\circ} \mathrm{C}$. vi) The membrane was removed from the solution and washed with Tris-buffered saline (TBS) three times for 5 min each time, after which a rabbit anti-mouse IgG secondary antibody (diluted 1:2500; Beijing Zhongshan Golden Bridge Biotechnology Co., Ltd., Beijing, China) was added and allowed to react at $37^{\circ} \mathrm{C}$ for $2 \mathrm{~h}$; then, the membrane was removed from the solution and washed with TBS once for $5 \mathrm{~min}$. vii) Enhanced chemiluminescence (ECL) staining (Pierce Protein Biology, Grand Island, NY, USA) was then applied to the membrane, and X-ray film was used for autoradiographic imaging, with GAPDH as the internal reference. The target bands were analyzed using Quantity One software.

Vector construction and transfection of polysialyltransferase constructs into cells. We constructed a lentiviral vector carrying ST8SiaII. The target vector was plv.ExBip/Neo-EF1a-ST8SIAII-eGFP, the control vector was plv.ExBip/Neo-EF1-eGFP, and the auxiliary vectors were plv/helper-SL3, plv/helper-SL4 and plv/helper-SL5. Meanwhile, RNA interference technology was used to select a polysialyltransferase ST8SiaII-shRNA plasmid for silencing of the expression of ST8SiaII in the SCLC H446 cells.

Transwell chamber invasion assay. The following procedure was used for the Transwell chamber invasion assay. i) As prepared according to the conventional 24-well Transwell chamber method, the upper chamber was lined with Matrigel and incubated at $37^{\circ} \mathrm{C}$ for $5 \mathrm{~h}$; then serum-free medium was used to gently wash the gel. ii) The post-treatment lung cancer cells from each group were collected and diluted with the RPMI-1640 serum-free medium to adjust the cellular concentration to $5 \times 10^{5} / \mathrm{ml}$, and $200 \mu \mathrm{l}$ of the cell suspension was added to the upper chamber, while $600 \mu \mathrm{l}$ of the cell culture medium (containing $5 \mu \mathrm{g} / \mathrm{ml}$ fibronectin) was added to the lower chamber. The chambers were cultured in a humidified incubator $\left(37^{\circ} \mathrm{C}\right.$ and $5 \%$ of $\left.\mathrm{CO}_{2}\right)$ for $24 \mathrm{~h}$. iii) Non-invasive cells were carefully wiped from the upper chamber. iv) The upper chamber was removed and placed upside down, fixed with $5 \%$ glutaraldehyde, and precooled at $4{ }^{\circ} \mathrm{C}$ for $30 \mathrm{~min}$. v) The cells were stained with an eosin solution and photographed; five visual fields were subjected to counting, and the mean value was used to quantify the transmembrane invasion of the lung cancer cells; the relative multiples were compared with the control H446 cell group.

The wound healing (cell metastasis) assay. After 48-72 h of culture, the cells of each group were harvested with trypsin-EDTA, the cell count was adjusted to $5 \times 10^{5} / \mathrm{ml}$, and the cells were seeded in 12-well plates and cultured overnight. After a monolayer formed, a sterile pipette tip was used to make scratches, and the cells were immediately photographed. The cells were then cultured for 24 or $48 \mathrm{~h}$, and were then photographed. The ImageJ software was used to quantitatively analyze the pixels in the scratched area, and the relative speed of cell metastasis was compared with that in the H446 cell group.

Statistical analysis. The data were analyzed using the SPSS 13.0 statistical package (SPSS for Windows; SPSS, Inc., Chicago, IL, USA), and the experiments were performed at least three times. The intergroup differences were analyzed with one-way analysis of variance (ANOVA) or the $\chi^{2}$ test; differences with $\mathrm{P}<0.05$ were considered statistically significant (very significant at $\mathrm{P}<0.01$ ).

\section{Results}

Expression of polysialylated NCAM and polysialyltransferases in SCLC cells. In the SCLC H446 cells, the results of IF revealed obvious expression of PSA-NCAM, manifested as green fluorescence (Fig. 1). The RT-PCR results at different annealing temperatures showed that the expression of ST8SiaII in every case was significantly stronger than that of ST8SiaIV, and the difference was significant $(\mathrm{P}<0.05)$. The LSCM results also confirmed significant expression of ST8SiaII in the H446 cells (Fig. 2).

ST8SiaII expression in different transfection groups. On the basis of the above results, we constructed the lentiviral vector carrying ST8SiaII. After construction of other recombinant vectors, the Lipofectamine 2000-cotransfected lentivirus was used to package the cells, and $48 \mathrm{~h}$ later, significant green fluorescence appeared, indicating that the transfection efficiency was good. The packaged viral particles and the prepared shRNA plasmid were then transfected into the target H446 cells. After repeated screening, a cell line that could stably and strongly express ST8SiaII was then established (H446-ST8SiaII-LENTI), as was the cell line (H446-ST8SiaII-shRNA) that showed stable suppression of ST8SiaII expression (Figs. 3 and 4).

Invasion and metastasis. The in vitro invasion and metastasis abilities of the three groups were tested. The Transwell assay showed that the transmembrane invasion ability of the H446-ST8SiaII-LENTI cell group was significantly increased as compared to the control group $(\mathrm{P}<0.01)$, while the in vitro transmembrane invasion ability of the H446-ST8SiaII-shRNA group was significantly inhibited compared to the control group ( $\mathrm{P}<0.01$; Fig. 5A). These results suggested that alterations of the expression of polysialyltransferase ST8SiaII can affect the post-transcriptional PSA modification of NCAM, thus having significant effects on the in vitro invasion ability of SCLC cells.

The in vitro metastasis potential of the three cellular groups was also examined to study the effects of alterations of the post-transcriptional PSA modification on the metastatic potential of SCLC. The wound healing assay revealed that the metastasis of the H446-ST8SiaII-LENTI cell group was significantly enhanced, while that of the H446-ST8SiaII-shRNA group was significantly inhibited ( $\mathrm{P}<0.01$ for both; Fig. 5B). These results suggested that alterations in the expression of polysialyltransferase ST8SiaII affected its capacity for the 

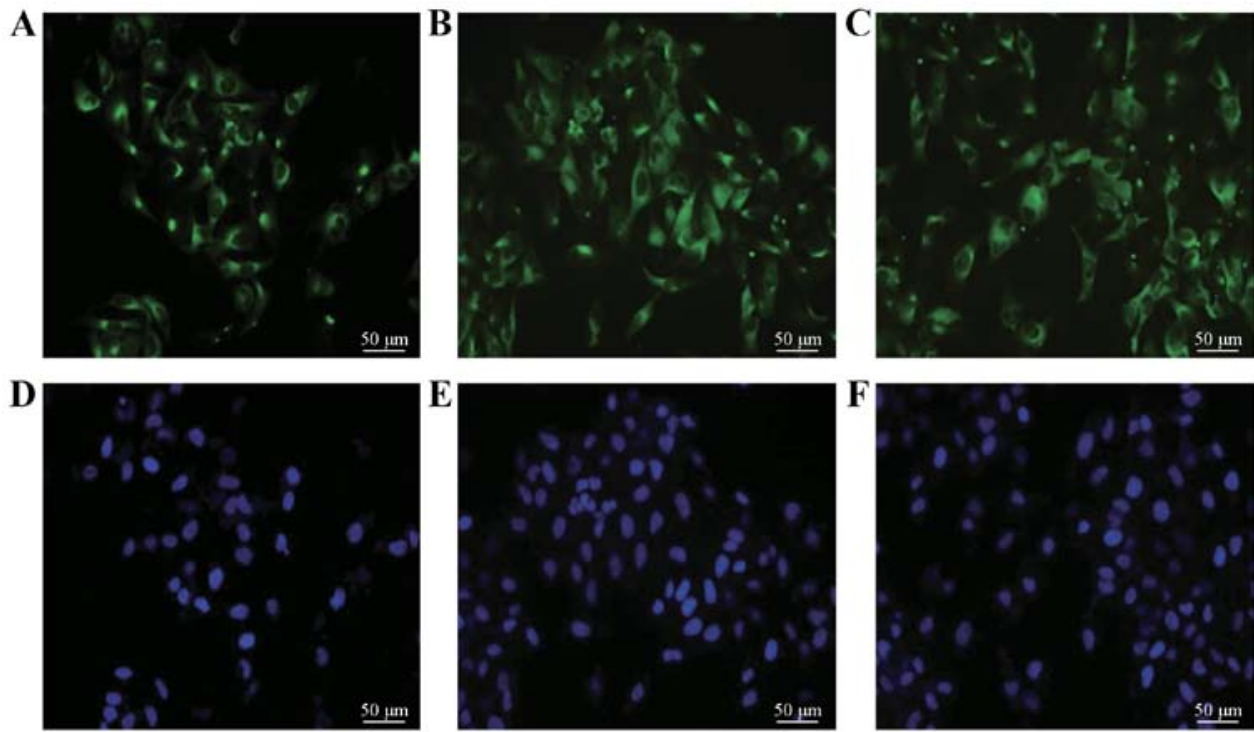

Figure 1. Expression of PSA-NCAM in the SCLC H446 cells (magnification, x200). (A-C) Immunofluorescent results of the PSA-NCAM-positive cells (green). (D-F) Nuclear staining (DAPI, blue)
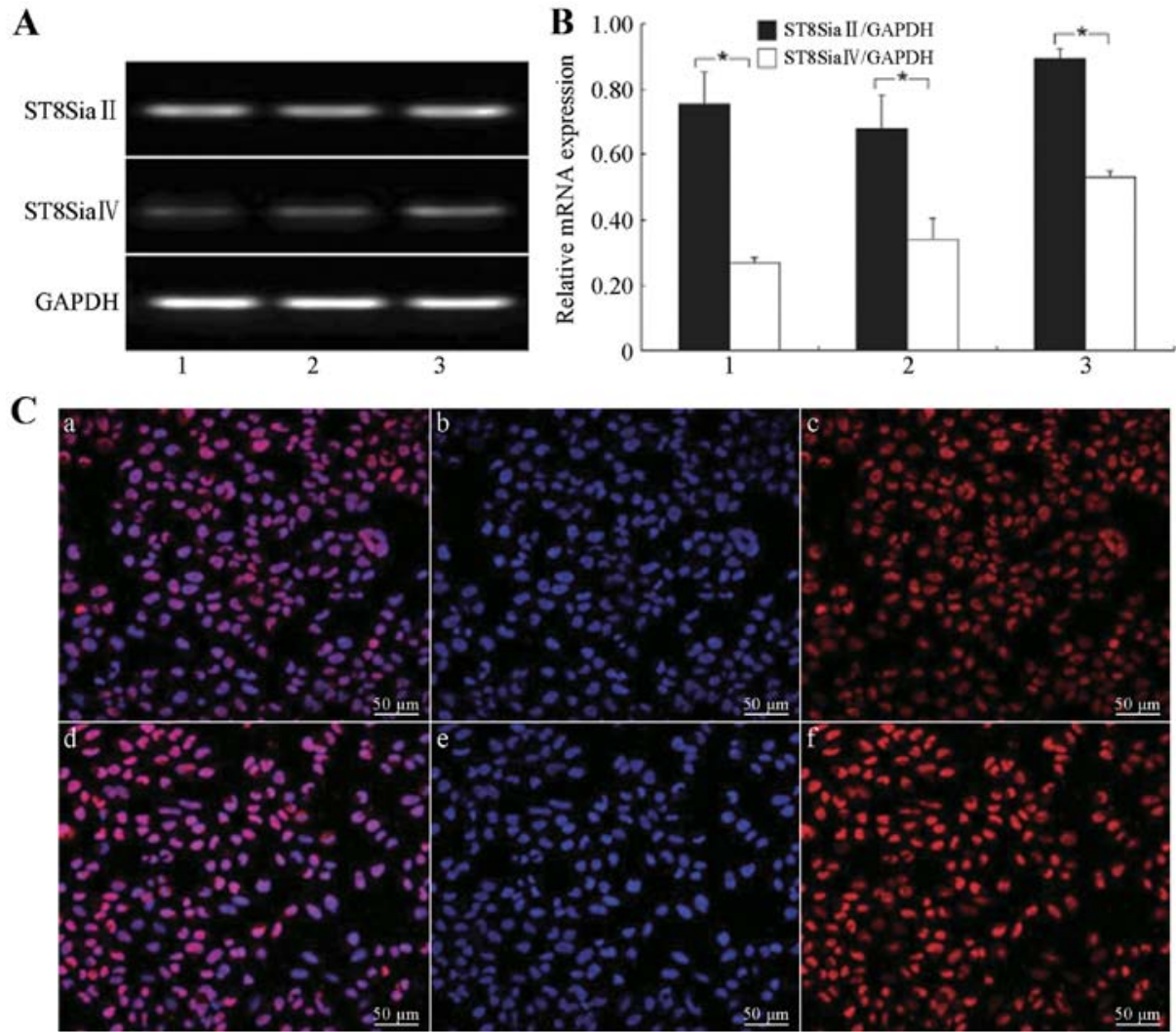

Figure 2. Expression levels of polysialyltransferase ST8SiaII and ST8SiaIV in the H446 cells. (A) Expression levels of polysialyltransferase ST8SiaII and ST8SiaIV in the $\mathrm{H} 446$ cells at different annealing temperatures: lane $1,56^{\circ} \mathrm{C}$; lane $2,59^{\circ} \mathrm{C}$; and lane $3,63^{\circ} \mathrm{C}$. (B) Relative mRNA expression levels of polysialyltransferase ST8SiaII and ST8SiaIV in the $\mathrm{H} 446$ cells $(" \mathrm{P}<0.05): 1,56^{\circ} \mathrm{C} ; 2,59^{\circ} \mathrm{C}$; and $3,63^{\circ} \mathrm{C}$. (C) LSCM detection of expression of polysialyltransferase ST8SiaII in the H446 cells: a and d, overlay of the ST8SiaII-positive and nuclear staining images; b and e, nuclear staining of H446 cells (DAPI, blue); $\mathrm{c}$ and f, ST8SiaII-positive cells (Cy3 staining, red, exciting wavelength $561 \mathrm{~nm}$ ). Scale bar, $50 \mu \mathrm{m}$.

post-transcriptional PSA modification of NCAM, thus influencing the in vitro metastasis of SCLC.

Changes in metastasis-related genes and signaling molecules. Western blot analysis was performed to detect the effects of the different treatments on SCLC metastasis-related genes. We found (Fig. 6A) that when the lentiviral transfection enhanced the expression of the ST8SiaII gene in the SCLC cells and affected the post-transcriptional PSA modification of NCAM (H446-ST8SiaII-LENTI), the protein expression 


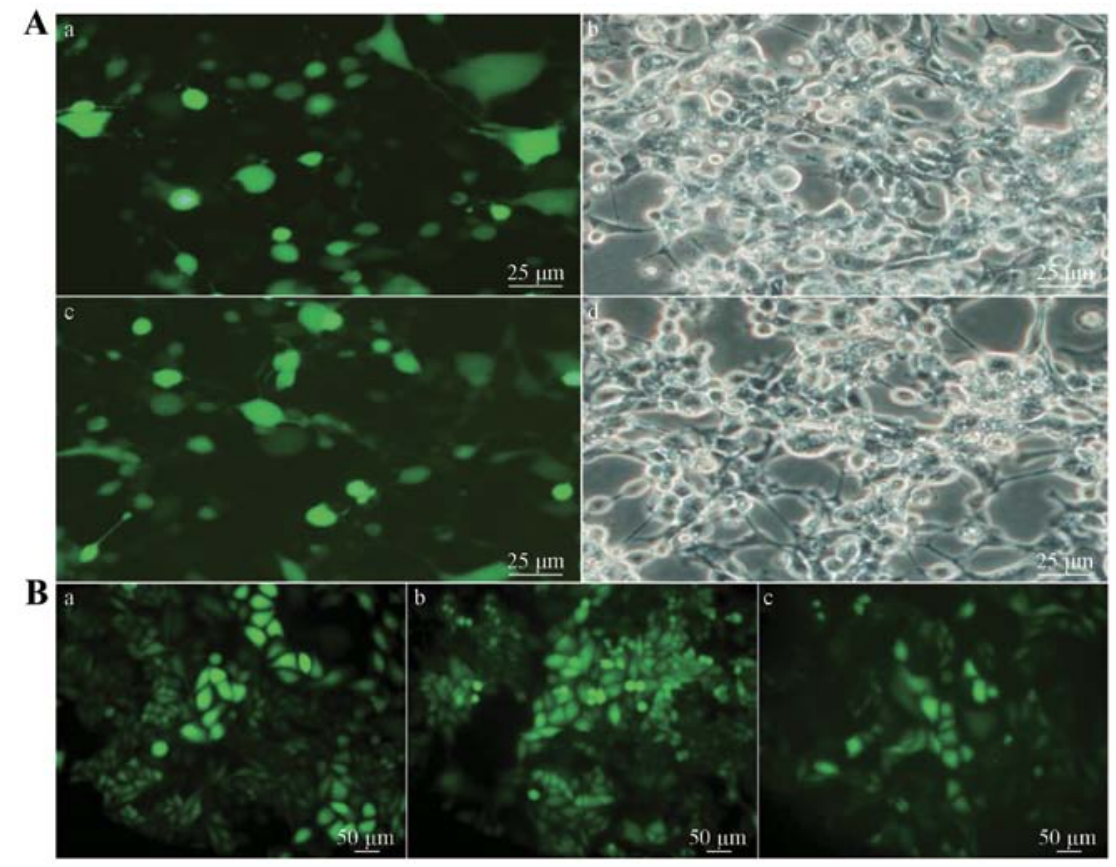

Figure 3. ST8SiaII lentiviral expression vectors, shRNA construction interference and cell transfection. (A) Transfection of recombinant vector liposome and lentiviral packaging: a and b, PLV/EGFP/ST8SiaII; -c and d, PLV/EGFP/NEO. (B) Transfection of lentiviral particles and shRNA plasmid into the target H446 cells: a, LENTI-EF1A-EGFP-control; b, LENTI-EF1A-EGFP-ST8SiaII; and c, PGFP-V-RS-ST8SiaII-shRNA.
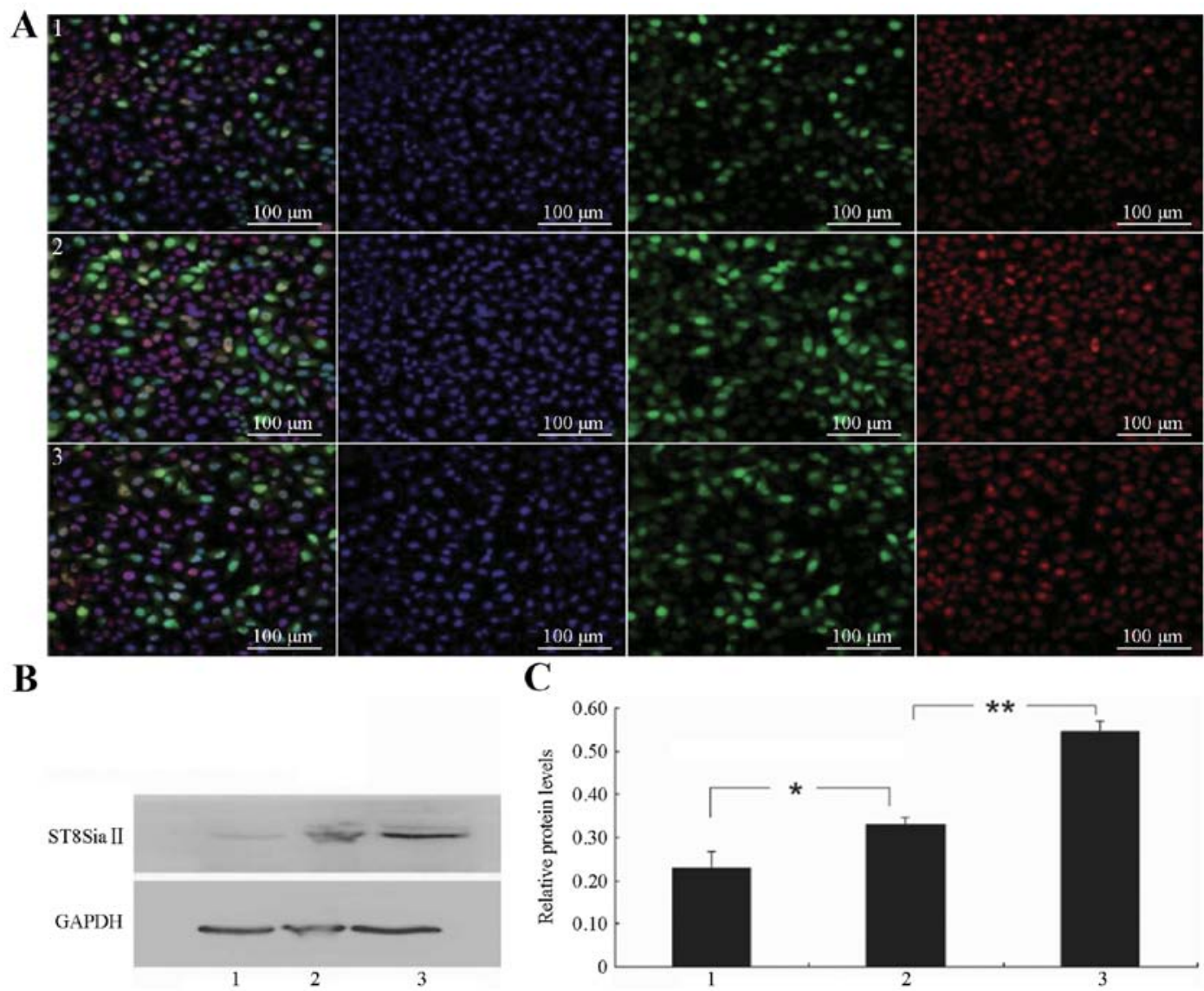

C

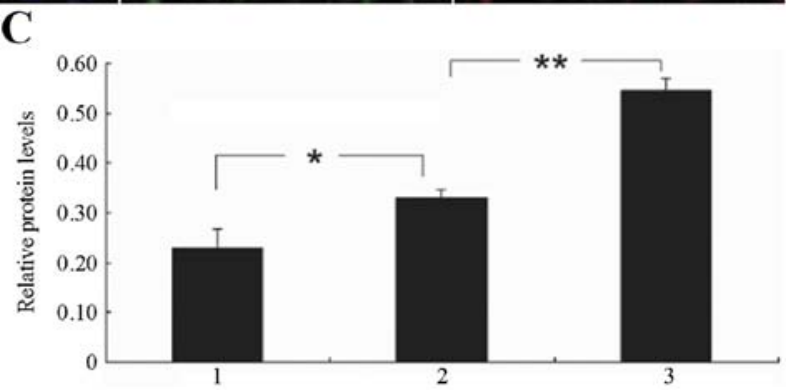

Figure 4. Expression of ST8SiaII in the different SCLC groups. (A) LSCM detection of ST8SiaII expression in the different transfection groups; 1-3 (left) overlay of the ST8SiaII-positive and nuclear staining images; (center left) nuclear staining of SCLC cells (DAPI, blue); (center right) the ST8SiaII-positive cells in the different groups (green, FITC staining, exciting wavelength $488 \mathrm{~nm}$ ); (right) the ST8SiaII-positive cells in the different groups (red, Cy3 staining, exciting wavelength $561 \mathrm{~nm}$ ). Scale bar, $100 \mu \mathrm{m}$. (B) Western blot detection of ST8SiaII protein expression in the different transfection groups. (C) Relative levels of ST8SiaII protein expression in the different transfection groups: 1, H446-ST8SiaII-shRNA; 2, H446 blank control; and 3, H446-ST8SiaII-LENTI; "P<0.05, ${ }^{* *} \mathrm{P}<0.01$ 
A
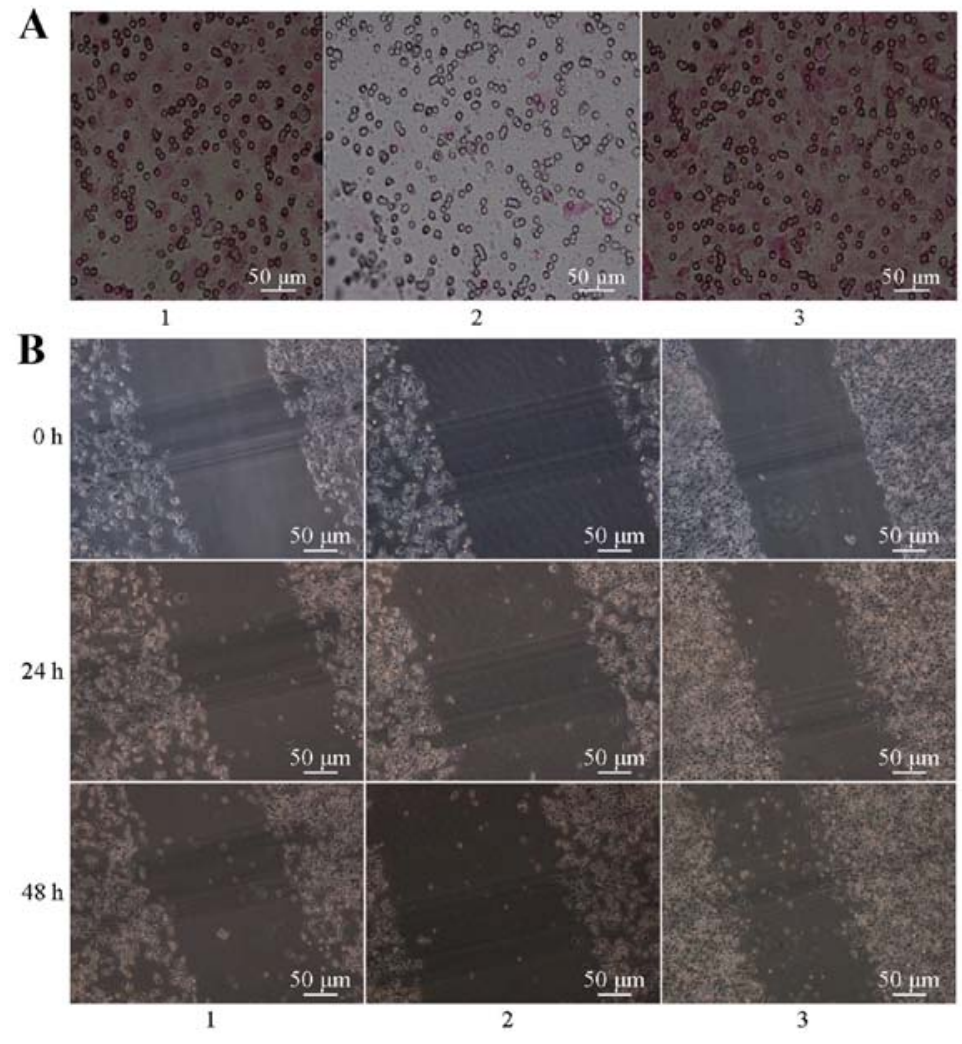
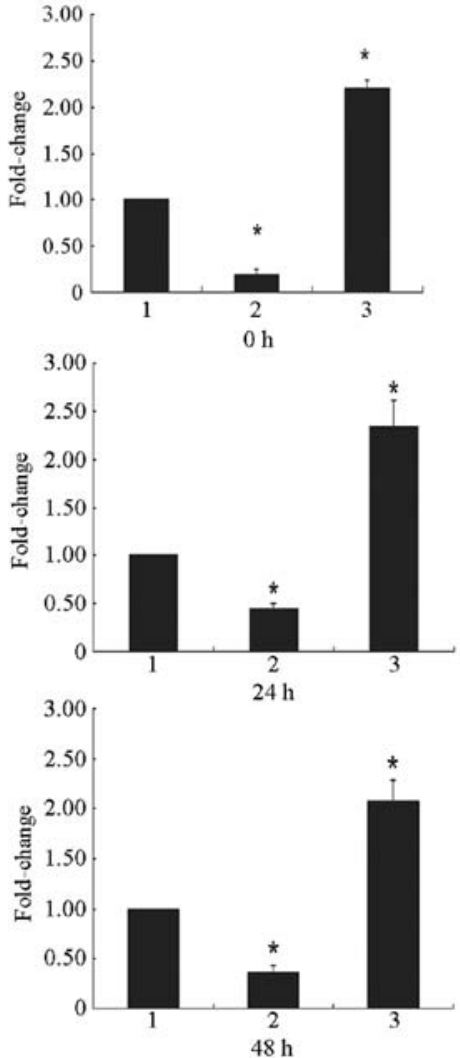

Figure 5. Impacts of polysialyltransferase regulation on the in vitro invasion and metastasis of SCLC. (A) Changes in the invasive ability of each group as detected by Transwell analysis (feosin, magnification, $\mathrm{x} 400$ ). (B) Changes in the metastasis of each group as detected by the wound healing analysis: 1 , H446 blank control; 2, H446-ST8SiaII-shRNA; and 3, H446-ST8SiaII-LENTI; ${ }^{*} \mathrm{P}<0.01$; scale bar, $50 \mu \mathrm{m}$.
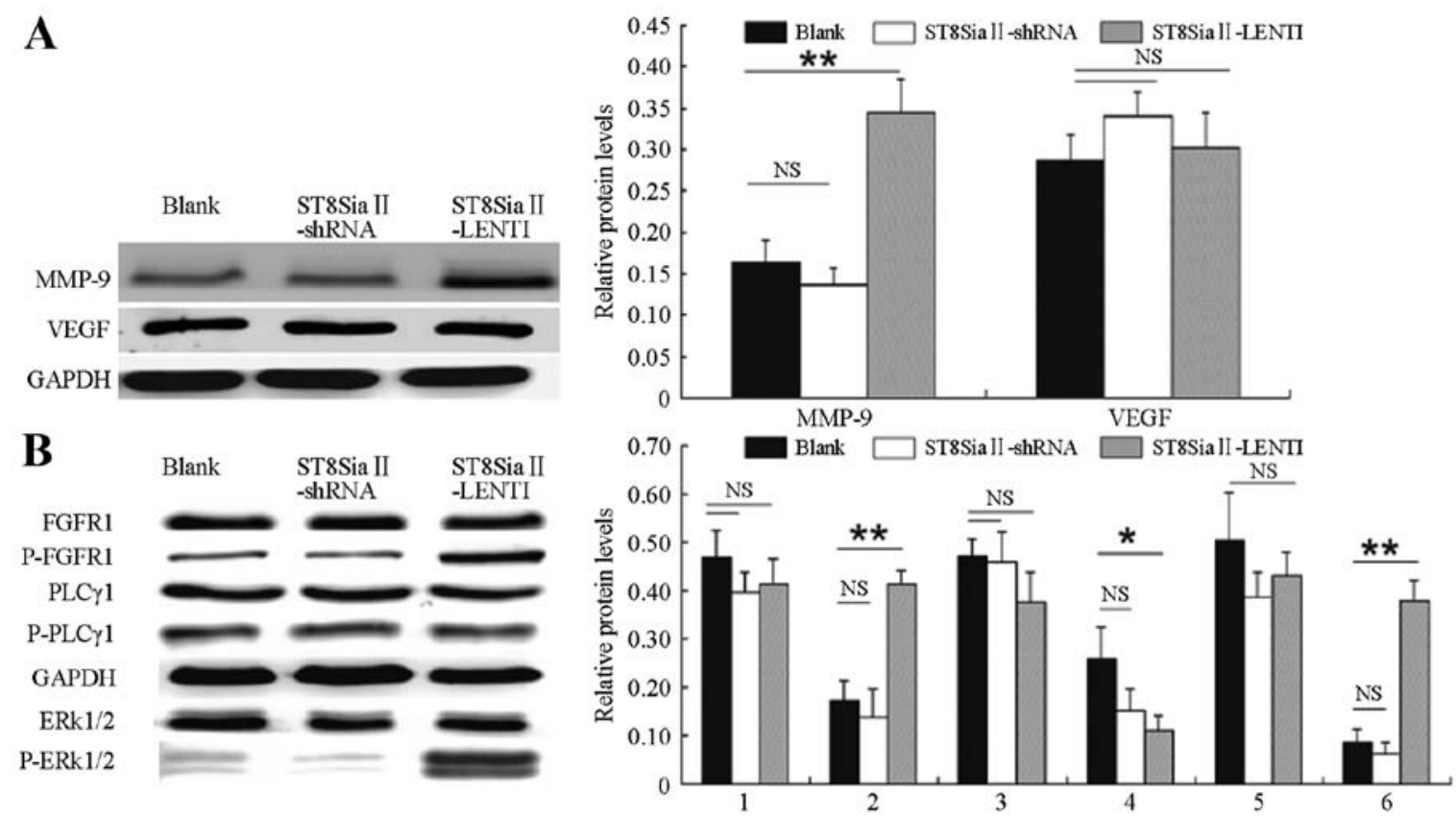

Figure 6. Changes in SCLC metastasis-related genes and signaling pathway molecules among the different groups. (A) Protein expression levels of MMP-9 and VEGF. (B) Protein expression and phosphorylation levels of FGFR, PLC $\gamma$ and ERK (1/2) among the experimental groups; 1, FGFR1; 2, P-FGFR1; 3, PLC $\gamma 1$; 4, P-PLC $\gamma 1 ; 5$, ERK (1/2); and 6, P-ERK (1/2); ${ }^{*} \mathrm{P}<0.05,{ }^{* *} \mathrm{P}<0.01$; NS, not significant.

levels of an invasion- and metastasis-related gene (MMP-9) was significantly increased $(\mathrm{P}<0.01)$, while the expression of $V E G F$ showed no statistically significant change when compared with the control group ( $\mathrm{P}>0.05)$. In addition, in the cells in which ST8SiaII was inhibited in a sustained fashion (H446-ST8SiaII-shRNA), the protein expression of MMP-9 
and VEGF showed no significant differences with the control group $(\mathrm{P}>0.05)$.

The protein expression and phosphorylation levels of intracellular signaling molecules were then further tested after treatment with various factors. The results (Fig. 6B) showed that in the H446-ST8SiaII-LENTI group, the protein expression levels of FGFR1, PLC $\gamma 1$ and ERK1/2 did not significantly differ from levels in the control group $(\mathrm{P}>0.05)$. In contrast, the expression levels of phosphorylated FGFR1 and ERK1/2 were significantly increased $(\mathrm{P}<0.01$ for both). The expression level of phosphorylated PLC $\gamma 1$ was decreased when compared with the level in the control group $(\mathrm{P}<0.05)$. In the H446ST8SiaII-shRNA group, the expression and phosphorylation levels of the above proteins showed no significant differences with the control group $(\mathrm{P}>0.05)$.

\section{Discussion}

Invasiveness and metastasis of SCLC seriously affect patient prognosis, but since the specific mechanisms have not been fully elucidated yet, the treatment effectiveness remains unsatisfactory. Active research into (and improved understanding of) the mechanisms of metastasis of SCLC and identification of new therapeutic targets will have a positive influence on the prevention and treatment of SCLC.

In the present study, we found that glycosylation of proteins or lipids on the tumor cell surface had significant effects on the metastatic potential of the tumor cells. The glycans are a class of biological molecules within cells, in addition to nucleic acids, proteins and lipids, and mainly include $\mathrm{N}$-glycans that are covalently linked to asparagine, O-glycans that are linked to serine or threonine, GAGs that are linked to serine, and glycolipids that are linked to lipid structures. Many of the body's physiological and pathological processes including inflammation, cancer, metabolic regulation, development, aging and immune defense are closely related to cellular glycans (11). PSA is a long linear chain formed by sialic acid monosaccharide residues ( $\mathrm{N}$-acetylneuraminic acid, Neu5AC; or N-hydroxy-acetylneuraminic acid, Neu5GC) via $\alpha-2,8$ bonds (7). The PSA chain in mammalian tissues generally consists of 50-150 residues, while the longest chain detected is $>370$ sialic acid residues long. PSA first caught the attention of researchers in the context of its roles in post-transcriptional modification of NCAM (12).

NCAM is a transmembrane protein on the cell surface and performs important functions in the development of nerve tissues as well as adhesion and metastasis of neural cells in the brain. The extramembranous structure of NCAM is composed of five Ig homeodomains (IgI, IgII, IgIII, IgIV and IgV) and two type III fibronectins (FN1 and FN2); the two adjacent domains are linked via disulfide bonds (13). The polysialylation of NCAM is mainly performed by ST8SiaII and ST8SiaIV, which have their catalytic regions in the Golgi lumen, and only ST8SiaII and ST8SiaIV can attach PSA to NCAM. The above two enzymes can catalyze the reaction alone or have synergistic effects, but the exact mechanisms have not been fully elucidated (7-9). The binding sites for polysialyltransferase on NCAM are mainly located in IgV and FN1 $(14,15)$. NCAM2 is also called olfactory cell adhesion molecule (OCAM) or Rb-8 neural cell adhesion molecule (RNCAM). NCAM1 and
NCAM2 share overall sequence identity of $\sim 44 \%$, but their functions are not exactly the same, and NCAM-2 cannot be covalently linked to PSA (16).

The present study showed for the first time that when the SCLC H446 cells were transfected with a lentivirus and the expression of polysialyltransferase ST8SiaII was enhanced (followed by enhancement of the PSA modification of NCAM) the in vitro invasion and metastasis abilities of the H446 cells were significantly increase. Expression of the invasion- and metastasis-related $M M P-9$ gene was also significantly increased $(\mathrm{P}<0.01)$, while VEGF showed no significant change. After further examining the changes in signaling molecules, we found that when ST8SiaII expression was enhanced, the expression levels of FGFR1, PLC $\gamma 1$ and ERK1/2 were not significantly changes, while the levels of phosphorylated FGFR1 and ERK1/2 were significantly increased $(\mathrm{P}<0.01)$. When shRNA was used to knock down the expression of polysialyltransferase ST8SiaII, the in vitro invasion and metastasis abilities of the H446 cells were also suppressed. In a similar fashion, Al-Saraire et al found that when cytidine monophosphate was used to inhibit the expression of ST8SiaII in neuroblastoma (SH-SY5Y) and glioma cells (C6-ST8SiaII type) (this treatment secondarily inhibits the ST8SiaII-mediated polysialylation of NCAM) the migration ability of these two tumor cell types was significantly decreased (10). In contrast, the migration of ST8SiaII itself [as well as the colon cancer cells (DLD-1) and wild-type glioma cells (C6 wild-type) that showed no expression of PSA] was not affected (10).

Recent studies (17-19) showed that the post-transcriptional modification of NCAM by PSA does not only affect its adhesion function but also has a 'switch' effect on the NCAM signal transduction functions. Before modification by PSA, it is easy for NCAMs to form stable homological adhesion. In contrast, when NCAM is highly modified by PSA, the steric hindrance effects of the long-chain PSA (as well as the mutual repulsion arising within negatively charged PSAs) inhibit the homological adhesion among NCAMs. This change results not only in separation of cancer cells but also in activation of the NCAM-mediated intracellular signaling pathways.

Various studies have confirmed that activated NCAM can activate intracellular signaling pathways mainly in two ways. One is the receptor tyrosine kinase-dependent signaling pathway; specifically, NCAM can act on FGFR or tyrosine kinase receptor $\mathrm{B}$ (TrkB) on the adjacent cell membrane, thus activating the receptor tyrosine kinase and promoting the downstream signaling cascades (20-22). Li et al (23) reported that PSA-modified NCAM can bind to the CAM homology domain (CHD) on the adjacent film FGFR, thereby modulating the activities of FGFR and promoting the metastasis of NIH-3T3 cells and African green monkey kidney cells COS-7. This finding is related to the phosphorylation of PLC $\gamma$ and ERK1/2 in the FGFR downstream signaling pathways.

The other pathway that NCAM activates using intracellular signals is the non-receptor-dependent pathway, which is not mediated by FGF receptors (24-26). It causes focal adhesion kinase (FAK) to bind to the NCAM-Fyn complex directly via phosphorylation of intracellular tyrosine protein kinase-related Fyn and FAK, then continues to activate the downstream Ras/mitogen-activated protein kinase (MEK)/ERK or 
phosphoinositide 3-kinase (PI-3K) signaling pathways (24-26). The specific mechanisms of how the activated NCAM can activate the intracellular signal transduction inside SCLC cells are still unclear. Expression of NCAM is present in almost all tissues of SCLC, and the diagnosis of SCLC can be confirmed by the results of immunohistochemical analysis (27).

The present study showed that, in SCLC, the post-transcriptional modification of NCAM with PSA glycans is closely associated with the metastatic potential of SCLC cells. We also found that activation of intracellular P-FGFR/P-ERK 1/2/ MMP-9 signaling by PSA-NCAM may be a new mechanism that promotes the invasiveness and metastasis of SCLC. This approach requires the FGF receptors to be phosphorylated, but not via PLC $\gamma$; thus, there may be additional mechanisms connecting phosphorylation of the FGF receptor to the activation of ERK1/2 phosphorylation. Future studies should further examine how the saccharification of cell surface molecules can activate specific intracellular signal transduction pathways and how the intracellular signaling pathways driving PSA-NCAM-mediated invasion and metastasis are regulated in SCLC. These lines of inquiry may lead to new strategies for the prevention or treatment of SCLC.

\section{Acknowledgements}

The present study was supported by the National Natural Science Foundation of China under grant no. 81272596.

\section{References}

1. Inamura $\mathrm{K}$ and Ishikawa $\mathrm{Y}$ : Lung cancer progression and metastasis from the prognostic point of view. Clin Exp Metastasis 27: 389-397, 2010.

2. Neal JW, Gubens MA and Wakelee HA: Current management of small cell lung cancer. Clin Chest Med 32: 853-863, 2011.

3. Manapov F, Klöcking S, Niyazi M, Levitskiy V, Belka C, Hildebrandt G, Fietkau R and Klautke G: Primary tumor response to chemoradiotherapy in limited-disease small-cell lung cancer correlates with duration of brain-metastasis free survival. J Neurooncol 109: 309-314, 2012.

4. Kalemkerian GP: Advances in pharmacotherapy of small cell lung cancer. Expert Opin Pharmacother 15: 2385-2396, 2014.

5. Morabito A, Carillio G, Daniele G, Piccirillo MC, Montanino A, Costanzo R, Sandomenico C, Giordano P, Normanno N, Perrone F, et al: Treatment of small cell lung cancer. Crit Rev Oncol Hematol 91: 257-270, 2014.

6. Paulson JC and Rademacher C: Glycan terminator. Nat Struct Mol Biol 16: 1121-1122, 2009.

7. Janas T and Janas T: Membrane oligo- and polysialic acids. Biochim Biophys Acta 1808: 2923-2932, 2011.

8. Foley DA, Swartzentruber KG and Colley KJ: Identification of sequences in the polysialyltransferases ST8Sia II and ST8Sia IV that are required for the protein-specific polysialylation of the neural cell adhesion molecule, NCAM. J Biol Chem 284: 15505-15516, 2009.

9. Falconer RA, Errington RJ, Shnyder SD, Smith PJ and Patterson LH: Polysialyltransferase: A new target in metastatic cancer. Curr Cancer Drug Targets 12: 925-939, 2012.
10. Al-Saraireh YM, Sutherland M, Springett BR, Freiberger F, Ribeiro Morais G, Loadman PM, Errington RJ, Smith PJ, Fukuda M, Gerardy-Schahn R, et al: Pharmacological inhibition of polysialyltransferase ST8SiaII modulates tumour cell migration. PLoS One 8: e73366, 2013.

11. Dube DH and Bertozzi CR: Glycans in cancer and inflammation - potential for therapeutics and diagnostics. Nat Rev Drug Discov 4: 477-488, 2005.

12. Durbec $\mathrm{P}$ and Cremer H: Revisiting the function of PSA-NCAM in the nervous system. Mol Neurobiol 24: 53-64, 2001

13. Soroka V, Kasper C and Poulsen FM: Structural biology of NCAM. Adv Exp Med Biol 663: 3-22, 2010.

14. Sandi C: Stress, cognitive impairment and cell adhesion molecules. Nat Rev Neurosci 5: 917-930, 2004.

15. Thompson MG, Foley DA, Swartzentruber KG and Colley KJ: Sequences at the interface of the fifth immunoglobulin domain and first fibronectin type III repeat of the neural cell adhesion molecule are critical for its polysialylation. J Biol Chem 286: 4525-4534, 2011

16. Kulahin $\mathrm{N}$ and Walmod PS: The neural cell adhesion molecule NCAM2/OCAM/RNCAM, a close relative to NCAM. Adv Exp Med Biol 663: 403-420, 2010.

17. Mühlenhoff M, Rollenhagen M, Werneburg S, Gerardy-Schahn R and Hildebrandt H: Polysialic acid: Versatile modification of NCAM, SynCAM 1 and neuropilin-2. Neurochem Res 38: 1134-1143, 2013

18. Berois N and Osinaga E: Glycobiology of neuroblastoma: Impact on tumor behavior, prognosis, and therapeutic strategies. Front Oncol 4: 114, 2014

19. Colley KJ, Kitajima K and Sato C: Polysialic acid: Biosynthesis, novel functions and applications. Crit Rev Biochem Mol Biol 49: 498-532, 2014

20. Ćirović S, Vještica J, Mueller CA, Tatić S, Vasiljević J, Milenković S, Mueller GA and Marković-Lipkovski J: NCAM and FGFR1 coexpression and colocalization in renal tumors. Int J Clin Exp Pathol 7: 1402-1414, 2014.

21. Cassens C, Kleene R, Xiao MF, Friedrich C, Dityateva G, Schafer-Nielsen $\mathrm{C}$ and Schachner M: Binding of the receptor tyrosine kinase TrkB to the neural cell adhesion molecule (NCAM) regulates phosphorylation of NCAM and NCAM-dependent neurite outgrowth. J Biol Chem 285: 28959-28967, 2010.

22. Nong L, Yin G, Ren K, Tang J and Fan W: Periodic mechanical stress enhances rat chondrocyte area expansion and migration through Src-PLCgamma1-ERK1/2 signaling. Eur J Cell Biol 89: 705-711, 2010.

23. Li J, Dai G, Cheng YB, Qi X and Geng MY: Polysialylation promotes neural cell adhesion molecule-mediated cell migration in a fibroblast growth factor receptor-dependent manner, but independent of adhesion capability. Glycobiology 21: 1010-1018, 2011.

24. Dalva MB, McClelland AC and Kayser MS: Cell adhesion molecules: Signalling functions at the synapse. Nat Rev Neurosci 8: 206-220, 2007.

25. Chattopadhyaya B, Baho E, Huang ZJ, Schachner M and Di Cristo G: Neural cell adhesion molecule-mediated Fyn activation promotes GABAergic synapse maturation in postnatal mouse cortex. J Neurosci 33: 5957-5968, 2013.

26. Eggers K, Werneburg S, Schertzinger A, Abeln M, Schiff M, Scharenberg MA, Burkhardt $\mathrm{H}$, Mühlenhoff $M$ and Hildebrandt $\mathrm{H}$ : Polysialic acid controls NCAM signals at cell-cell contacts to regulate focal adhesion independent from FGF receptor activity. J Cell Sci 124: 3279-3291, 2011.

27. Teicher BA: Targets in small cell lung cancer. Biochem Pharmacol 87: 211-219, 2014. 